\title{
FAIT ET EIDOS : HUSSERL, MERLEAU-PONTY, RICHIR
}

\author{
CLAUDIA SERBAN
}

\begin{abstract}
The reworking and the renewal of the eidetic project and method is at the very heart of Marc Richir's phenomenology. Our paper examines his close reading of Husserl and Merleau-Ponty, paying attention to the directions that the German and the French phenomenologist assigned to the consideration of the eidos and to that of the distinction between essence and fact. After having identified, in Husserl, two senses of facticity that imply a different relation to the eidos, we focus on Merleau-Ponty's emphasis on the "indivision" between the essential and the factual, and on his conception of a wild Wesen or of a "raw Being (Etre brut)". At last and in connection with these previous elements of analysis, we provide an overview of Richir's main contributions to a phenomenological eidetics, highlighting, in particular, the importance that he attaches to the irreducible indeterminacy of the eidos, to its ever-unfinished genesis and to the impossibility of its totalization or its complete unification.
\end{abstract}

Au seuil de l'Appendice qui clôt les Méditations phénoménologiques (1992), Marc Richir souligne «l'importance stratégique du rapport entre fait et eidos pour l'architectonique de la phénoménologie $»^{1}$, pour relever aussitôt la fécondité de sa transposition ou de sa compréhension en termes de « rapport entre facticité et essentialité », en renvoyant au concept merleau-pontien de Wesen sauvage. Pourtant, comme nous le savons, cet Appendice important est consacré à Husserl, et plus précisément, à un texte tardif de Husserl composé de notes datées de novembre 1931 et édité par Iso Kern en 1973 dans le volume XV des Husserliana,

1 Richir Marc, Méditations phénoménologiques, Grenoble, Jérôme Millon, 1992, p. 380. 
texte intitulé « Téléologie » et sous-titré par son éditeur : «L'implication de l'eidos intersubjectivité transcendantale dans l'eidos Moi transcendantal. Fait et eidos $»^{2}$. La signification particulière de ce manuscrit de travail provient de l'articulation remarquable et complexe qu'il opère, non seulement entre facticité et essentialité, mais aussi, plus précisément, entre facticité, eidétique, subjectivité et intersubjectivité, sous le signe du problème de la téléologie. Husserl y retrace en effet le processus de la constitution de l'eidos, ce qui le conduit vers une dimension rarement aperçue par lui avant le début des années trente : celle d'une facticité d'un type nouveau, la facticité de l'ego qui, en tant que telle, vaut comme facticité de l'absolu et nous confronte ainsi à une dimension nouvelle et inattendue de l'absolu phénoménologique. À ce propos, Richir note que si, d'une part, au terme du processus de variation qui délivre l'essence en tant qu'invariant, la réduction eidétique procède à l' " élision de la facticité " ${ }^{3}$, d'autre part - comme Merleau-Ponty se plaisait à le rappeler en soulignant, dès l'Avant-propos de la Phénoménologie de la perception jusqu'au Visible et l'invisible, l'intime solidarité entre réduction eidétique et réduction transcendantale - l'eidétique, en tant qu'elle résulte d'un processus de variation, possède inévitablement quelque chose comme une "matrice transcendantale $»^{4}$. Or c'est précisément en vertu de cette matrice transcendantale de l'eidétique qu'il y a selon Richir, comme le Forschungsmanuskript husserlien intitulé « Téléologie » le montre, " ancrage des possibilités eidétiques [...] et de l'eidos dans ma facticité »; et plus loin encore, "si l'eidos est construit en moi, dans ma facticité, il l'est aussi dans les autres, dans leur facticité ; les autres ne sont pas moins constituants que moi-même $»^{5}$. Autrement dit, c'est au niveau de cette facticité égologique ultime, en tant que soubassement le plus profond de la matrice transcendantale de l'eidétique, que s'ouvre la dimension de l'interfacticité : la considération décisive de l'interfacticité doit compléter ainsi celle de l'intersubjectivité transcendantale.

Il est donc légitime à plusieurs titres de parler ici, comme le fait Richir dans l'Appendice " Fait et eidos", d'un "changement architectonique de registre » : car non seulement cette " coalescence de la facticité et de l'essentialité " ${ }^{6}$ est à même d'exhiber l'assise ultime de l'édifice eidétique, mais elle remanie aussi de part en part le sens de la facticité égologique ou subjective, jusqu'à nous placer au niveau d'une interfacticité qui exprime sans doute la vérité ultime de l'intersubjectivité

2 Husserl Edmund, Zur Phänomenologie der Intersubjektivität. Texte aus dem Nachlass, Dritter Teil : 1929-1935, Husserliana, tome XV, éd. par Iso Kern, La Haye, Nijhoff, 1973, texte n²2, p. 378-386.

Richir M., Méditations..., op. cit., p. 383.

Richir M., Méditations..., op. cit., p. 385.

Richir M., Méditations..., op. cit., p. 384.

Richir M., Méditations..., op. cit., p. 385 et 386. 
transcendantale. Et Richir de conclure, en ressaisissant de manière synthétique l'enseignement fondamental du texte de Husserl : "C'est donc la facticité du moi et de mon monde, et la facticité intersubjective, c'est-à-dire l'interfacticité [...] qui font toute la téléologie de l'institution symbolique se faisant. " ${ }^{7}$ Cette leçon décisive est prolongée selon une grille d'interprétation particulière : en faisant apparaitre la coïncidence de la facticité et de la nécessité, Husserl procéderait en dernière instance à un "remaniement [...] de l'onto-théologie " tout en nous livrant sa propre version d'une ontologie fondamentale ${ }^{8}$. Cependant, si le texte husserlien paraît nous inviter à distinguer, comme le souligne Richir, entre " la facticité divine originaire, et absolument nécessaire, et notre facticité $»^{9}$, il le fait sur un mode simplement interrogatif, dans un passage que le phénoménologue belge qualifie d' " abyssal »: " Peut-on dire, dans cette situation que cette téléologie, avec sa facticité originaire, a son fondement (Grund) en Dieu ? » ${ }^{10}$ Cette occurrence de Dieu dans le texte demeure néanmoins un hapax qui sert à poser la question de l'absolu phénoménologique dans toute son abyssalité, sans décider pour autant que cet absolu soit d'une nature autre qu'égologique, voire proprement divine. Mais sans vouloir ouvrir ici le dossier complexe de la réappropriation husserlienne de la question théologique ${ }^{11}$, nous nous contenterons de relever ce qui nous paraît être la critique la plus puissante formulée par Richir au terme de sa lecture du manuscrit intitulé " Téléologie » : « Husserl n'arrive pas, très significativement, à penser tout Wesen comme porté intrinsèquement par la facticité, il arrive seulement à penser la facticité de l'eidétique prise dans son ensemble. »12

Cette formulation de l'insuffisance de la position de Husserl suggère, plus que ne le dit expressément l'Appendice des Méditations phénoménologiques, qu'il y aurait chez Husserl plusieurs régimes eidétiques et plusieurs façons de concevoir le rapport entre fait et eidos, de même que - et cela se trouve plus explicitement souligné par Richir - l'ordre des faits est lui-même scindé entre, d'une part, « la factualité des faits mondains dans leur Vorhandenheit » et, d'autre part, « la facticité et [...] l'interfacticité mises en jeu par la certitude factice d'exister du cogito » ${ }^{13}$.

7 Richir M., Méditations..., op. cit., p. 386.

8 Richir M., Méditations..., op. cit., p. 388 et 387.

9 Richir M., Méditations..., op. cit., p. 389.

10 Husserl E., Zur Phänomenologie..., op. cit., p. 385, cité dans Richir M., Méditations..., op. cit., p. 386.

11 À ce sujet, nous renvoyons notamment aux textes publiés en 2014 au tome XLII des Husserliana (Grenzprobleme der Phänomenologie. Analysen des Unbewusstseins und der Instinkte. Metaphysik. Späte Ethik (Texte aus dem Nachlass 1908-1937), éd. par Rochus Sowa et Thomas Vongehr, New York: Springer), ainsi qu'à l'analyse d'Emmanuel Housset dans Husserl et l'idée de Dieu (Paris, Cerf, 2010).

12 Richir M., Méditations..., op. cit., p. 388.

13 Richir M., Méditations..., op. cit., p. 387. 
Nous nous proposons dès lors de montrer la légitimité et la nécessité de distinguer entre un régime, pour ainsi dire, commun et un régime égologique de l'eidétique dans la phénoménologie de Husserl ; ce sera le premier temps de notre propos. Notre deuxième objectif consistera à revenir rapidement, en suivant l'impulsion donnée par la référence tutélaire au concept de Wesen sauvage, sur la refonte merleau-pontienne de l'eidétique, afin de voir dans quelle mesure elle échappe à la critique adressée par Richir à Husserl et réussit, pour sa part, à " penser tout Wesen comme porté intrinsèquement par la facticité ». Enfin, dans un dernier moment, nous nous proposons de passer en revue les jalons ultérieurs de la reprise de la question eidétique par l'auteur des Méditations phénoménologiques, notamment dans L'institution de l'idéalité (2002) et dans les Fragments phénoménologiques sur le langage (2008), sans oublier de rappeler le dialogue fécond avec l'auteur du Visible et l'invisible dans Phénomènes Temps et Etres (1987). Ce parcours essaiera aussi d'apporter un éclairage sur la signification que Richir attribue au concept de Wesen sauvage, dont la résonance merleau-pontienne ne dévoile pas toute la portée.

\section{Husserl : I'eidétique et le statut de la facticité}

Nous commencerons donc par confronter les deux formes que prend l'eidétique chez Husserl - l'eidétique des objectités (fussent-elles réales ou idéales), d'une part, et l'eidétique égologique de l'autre - à l'aune de la question suivante : l'eidos a-t-il le même statut et, surtout, le même rapport au fait et à la facticité dans les deux cas ? Nous pouvons partir à ce dessein de l'analyse des rapports entre fait et eidos que l'on trouve au début des Ideen I et qui pose une forte relation de dépendance entre fait et essence, en vertu du caractère d'acte fondé de l'intuition eidétique. Le $\$ 3$ souligne, en effet, que l'intuition de l'essence ne peut pas se déployer là où une intuition de l'individu fait complètement défaut. Seulement, on aurait tort de vouloir interpréter cette thèse au sens d'un asservissement de la connaissance eidétique par rapport à la connaissance factuelle, ou comme position d'une dépendance de l'essence à l'égard d'une quelconque existence. Car l'individu dont on a besoin, à titre d'exemple, pour s'élever vers l'eidos, peut être cherché dans le domaine du simple possible aussi bien que dans celui de l'effectif ${ }^{14}$. Le possible qui peut servir de point de départ à la mise au jour de l'essence est ici

14 Husserl E., Ideen zu einer reinen Phänomenologie und phänomenologischen Philosophie, livre premier : Allgemeine Einführung in die Phänomenologie, Husserliana, tome III/1,éd. par Karl Schuhmann, La Haye, Nijhoff, 1976, p. 16 ; trad. fr. Idées directrices pour une phénoménologie et une 
l'imaginaire, le produit de la Phantasie : c'est donc à cet endroit que s'amorce le rôle insigne de la fiction pour la recherche phénoménologique - fiction dont Husserl dira plus loin (au $\$ 70$ des Ideen I) qu'elle est «l'élément vital de la phénoménologie comme de toutes les sciences eidétiques ${ }^{15}$. En ce sens, Husserl notait déjà en 1907, dans les leçons qui ont été publiées sous le titre L'idée de la phénoménologie: "Pour l'étude de l'essence, la perception (Wahrnehmung) et la représentation imaginaire (Phantasievorstellung) sont tout à fait sur le même plan. ${ }^{16}$

Par cette libération de la connaissance eidétique à l'égard des faits se produit ainsi un renversement de la supposée fondation ou dépendance de l'essence. Le ressort de cette émancipation de l'eidétique n'est autre que l'importance croissante de l'imagination comme voie d'accès aux possibilités. En effet, la Phantasie peut fournir l'exemple qui va servir à la mise en place de l'eidos, en évitant le détour contraignant par les faits et par l'expérience des effectivités : à cette contrainte immaitrisable elle substitue la liberté de la fiction, et c'est pourquoi, au bout du compte, Husserl peut affirmer qu' « en phénoménologie comme dans toutes les sciences eidétiques, les présentifications, et pour parler plus précisément, les images libres ont une position privilégiée par rapport aux perceptions $\gg{ }^{17}$. Cette avancée constitue une relativisation non négligeable des droits de la Leibhaftigkeit, de la présence en chair et en os, tels qu'ils sont détenus par la perception : plus importante et plus forte que l'exigence de Leibhaftigkeit est la considération du pouvoir de variation et de l'extension du domaine des possibilités que l'imagination est susceptible d'ouvrir, là où la perception se borne à présenter l'effectif singulier. La Phantasie est ainsi essentiellement synonyme de liberté, de «l'incomparable liberté » qui donne « accès au champ immense des possibilités eidétiques ainsi qu'aux connaissances eidétiques qui leur font un horizon infini ${ }^{18}$.

Affirmer, de façon provocante et audacieuse, que « la fiction est la source où s'alimente la connaissance des 'vérités éternelles' » est donc aussi une façon de miner définitivement l'hégémonie empiriste du fait sur l'essence pour rétablir l'autonomie de l'ordre eidétique là où le caractère dérivé de la Wesenschau semblait suggérer une servitude. C'est à présent la connaissance factuelle qui nécessite d'être fondée dans la connaissance eidétique, et c'est pour cette raison que, de

philosophie phénoménologique pures, tome premier : Introduction générale à la phénoménologie pure, par Paul Ricœur, Paris, Gallimard, 1950, p. 24, trad. modif.

15 Husserl E., Ideen zu..., op. cit., p. 148 ; trad. fr., p. 227.

16 Husserl Edmund, Die Idee der Phänomenologie. Fünf Vorlesungen, Husserliana, tome II, éd. par Walter Biemel, La Haye, Nijhoff, 1950, p. 68 ; trad. fr. : L'idée de la phénoménologie. Cinq leçons, par Alexandre Lowit, Paris, PUF, 1970, p. 93.

17 Husserl E., Ideen zu..., op. cit., p. 147 ; trad. fr., p. 225.

18 Husserl E., Ideen zu..., op. cit., p. 147 ; trad. fr., p. 225. 
façon fort significative, Husserl n'hésite pas à réhabiliter dans les Ideen I «l'antique doctrine ontologique selon laquelle la connaissance du 'possible' doit précéder celle des effectivités ${ }^{19}$. Or la liberté de l'imagination peut aller jusqu'à briser insensiblement tout lien avec le monde factuel. La réduction eidétique, rigoureusement comprise et déployée, peut retirer ainsi tout privilège au monde existant et le situer au même niveau que les mondes simplement possibles, en les envisageant tous uniquement du point de vue de leur égale possibilité. Par conséquent, à ce niveau de l'eidétique que nous avons désigné précédemment comme son régime commun, plus haut que la facticité se tient la possibilité et, en ce sens, en tant qu'eidétique, la phénoménologie husserlienne peut être décrite comme une phénoménologie de la possibilité20.

Affirmer que la phénoménologie est une science ou une discipline eidétique, c'est donc dire aussi qu'elle n'est fondamentalement pas la science des faits ou de ce qui existe effectivement; et même, toutes les questions (ou du moins les " questions ultimes ») relatives aux faits, elle les réserve, comme le montre la célèbre conclusion des Méditations cartésiennes, à une autre discipline que Husserl qualifie sans hésiter de "métaphysique " et qui est censée prendre en compte les " problèmes de la facticité contingente (zufällige Faktizität), de la mort, du destin ${ }^{21}$, etc. De ce point de vue, la facticité n'est pas tant un problème phénoménologique qu'un problème métaphysique ; le phénoménologue peut, pour l'essentiel, se contenter de l'étude des possibilités eidétiques et réserver celle des effectivités au métaphysicien qui, selon une caractérisation que Husserl en donne dès 1908, « interroge en direction de 'l'effectivité au sens ultime' $»^{22}$.

Mais comme la fin des Méditations cartésiennes le suggère, il peut y avoir cependant, dans une certaine mesure, récupération, à l'intérieur de la phénoménologie, de ce qui se présente de prime abord comme factuel ou facticiel, même si cela ne revient pas à relativiser l'opposition entre fait et eidos, mais plutôt à la renforcer. La quatrième et dernière version de l'article " Phénoménologie » pour l'Encyclopaedia Britannica (1927) résume cette situation paradoxale en divisant de

19 Husserl E., Ideen zu..., op. cit., p. 178 ; trad. fr., p. 269.

20 Comme nous l'avons fait dans notre ouvrage éponyme (Paris, PUF, 2016).

21 Husserl E., Cartesianische Meditationen und Pariser Vorträge, Husserliana, tome I, éd. par Stephan Strasser, La Haye, Nijhoff, 1950, p. 39 et 182 ; trad. fr. : Méditations cartésiennes et les Conférences de Paris, par Marc de Launay, Paris, PUF, 1994, p. 40 et 208.

22 Husserl E., Transzendentaler Idealismus. Texte aus dem Nachlass (1908-1921), Husserliana, tome XXXVI, éd. par Robin D. Rollinger, Dordrecht, Springer, 2003, p. 22.

Nous avons abordé la question de la relation entre phénoménologie et métaphysique à l'aune des rapports entre le possible et l'effectif dans « Métaphysique de l'effectivité, métaphysique de la facticité : Le problème d'une métaphysique phénoménologique », in Phänomenologie \& Métaphysik, ed. par Inga Römer et Alexander Schnell, Phänomenologische Forschungen, 2020, p. 3-20. 
façon bien remarquable la phénoménologie transcendantale en une "philosophie première " (la phénoménologie eidétique) et une " philosophie seconde ${ }^{23}$ (la science de l'universum des facta) qui ressemble en un sens, comme son nom aristotélicien le laisse entendre, plus à une physique (au sens très large du terme) qu'à une métaphysique. Cette tentative de récupération de la facticité à l'intérieur du territoire phénoménologique ne va pourtant pas sans une nette subordination, peu surprenante d'ailleurs, de la science des faits à l'égard de l'eidétique : « La philosophie première est l'universum de la méthode pour la philosophie seconde $»^{24}$. L'approche de la facticité ne saurait, à ce niveau, se passer de l'eidos ou être indépendante de l'eidétique.

Il semble néanmoins y avoir un cas où ce privilège et cette primauté de l'eidos ne se laissent pas maintenir tels quels : ce cas est celui de l'ego lui-même, qui ne saurait être conçu indifféremment comme illustration d'une essence ou comme une existence donnée dans les faits. Cette réévaluation importante de la facticité de l'ego apparait de manière éclatante dans le manuscrit de travail de novembre 1931 commenté par Richir, où Husserl affirme expressément que la facticité est insurmontable et irréductible ("Mein faktisches Sein kann ich nicht überschreiten»), jusqu'à en inférer la dépendance de l'eidos ego à l'égard de l'ego facticiel ${ }^{25}$.

Le fait de l'ego vient ainsi avant l'eidos ego. Mais cela ne revient toutefois pas à annuler le clivage entre fait et essence : si c'était véritablement le cas, il n'y aurait plus vraiment de place pour l'alter ego, car l'eidos ego s'épuiserait dans le fait de mon ego. Plutôt qu'à l'éliminer, l'égologie invite à penser autrement l'écart de l'eidos par rapport au fait : là où l'eidétique de l'objet pouvait s'échapper, au moyen de l'imagination, vers le royaume des « pures possibilités » et permettait de n'envisager le fait «que comme factum de sa propre essence et par sa propre essence $»^{26}$, l'eidétique égologique exige de prendre plus au sérieux la pierre d'achoppement de la facticité. Et la lecture de Richir radicalise le geste husserlien à ce niveau précis, en exhibant en outre la nécessité de penser la facticité égologique comme interfacticité.

23 Husserl E., Phänomenologische Psychologie. Vorlesungen Sommersemester 1925, Husserliana, tome IX, éd. par Walter Biemel, La Haye, Nijhoff, 1962, p. 298 ; trad. fr. : Psychologie phénoménologique (1925-1928), par Philippe Cabestan, Natalie Depraz et Antonino Mazzù, Paris, Vrin, 2001, p. 241 et 242 .

24 Husserl E., Phänomenologische..., op. cit., p. 298-299; trad. fr., p. 242.

25 Husserl E., Zur Phänomenologie..., op. cit., p. 383-386.

26 Husserl E., Die Krisis der europäischen Wissenschaften und die transzendentale Phänomenologie. Eine Einleitung in die phänomenologische Philosophie, Husserliana, tome VI, éd. par Walter Biemel, La Haye, Nijhoff, 1954, p. 182 ; trad. fr. : La crise des sciences européennes et la phénoménologie transcendantale, par Gérard Granel, Paris, Gallimard, 1976, p. 203. 


\section{Merleau-Ponty et le Wesen sauvage comme essence brouillée par les faits}

Cette rapide traversée du projet eidétique de Husserl a permis d'étoffer quelque peu la critique que lui adressait Richir à la fin des Méditations phénoménologiques, critique selon laquelle il «n'arrive pas, très significativement, à penser tout Wesen comme porté intrinsèquement par la facticité $»^{27}$. Le moment est venu de voir dans quelle mesure Merleau-Ponty, convoqué par la généalogie du concept de Wesen sauvage ${ }^{28}$, a été à la hauteur de cette tâche, ce qui nous permettra aussi de décider de la signification à conférer à ce concept de Wesen sauvage dans un horizon strictement merleau-pontien. Il faut partir à ce dessein de sa réappropriation, remarquable à plus d'un titre, du projet eidétique husserlien. Nous avons déjà rappelé que, dès le fameux Avant-propos à la Phénoménologie de la perception, Merleau-Ponty insiste sur l'intrication étroite entre réduction transcendantale et réduction eidétique ${ }^{29}$. Or ce faisant, il accorde aussitôt à l' eidétique une signification assez peu conforme au vœu de Husserl, en affirmant qu' « il est clair que l'essence n'est pas ici le but, qu'elle est un moyen » et que « la nécessité de passer par les essences » se fonde dans le besoin qu'a notre existence « du champ de l'idéalité pour connaître et conquérir sa facticité ${ }^{30}$. Merleau-Ponty opère donc à bon escient une certaine déformation de la phénoménologie husserlienne afin de la mettre à l'abri des critiques qui y voient un nouvel essentialisme déconnecté de la considération des faits ou de l'existence : à ses yeux, la méthode phénoménologique mise en œuvre par Husserl ne défait jamais le "nœud de l'essence et de l'existence », car le phénomène central sur lequel elle demeure orientée est celui du "sens incarné ", sens qui «ne fait qu'un avec l'existence ${ }^{31}$. Dès lors, il lui est possible d'avancer, dans le même Avant-propos, que «la méthode eidétique est celle d'un positivisme phénoménologique qui fonde le possible sur le réel ${ }^{32}$. Ce faisant, Merleau-Ponty refuse la prééminence que Husserl accordait au possible au sein de son projet eidétique et, plus profondément encore, récuse la compréhension de ce projet sous

\footnotetext{
Richir M., Méditations..., op. cit., p. 388.

28 Florian Forestier se penche rapidement sur cette filiation dans son ouvrage La phénoménologie génétique de Marc Richir, Dordrecht, Springer, 2015, p. 23-25, mais considère que Merleau-Ponty reste encore tributaire d'une quête du logos et de l'originaire et estime dès lors plus pertinent de rapprocher le concept richirien de Wesen sauvage de Derrida et de sa pensée de «l'archi-écriture ».

29 Merleau-Ponty Maurice, Phénoménologie de la perception, Paris, Gallimard, 2005 [1945], p. 15. Voir aussi Merleau-Ponty M., Le visible et l'invisible, Paris, Gallimard, 1997 [1964], p. 70.

30 Merleau-Ponty M., Phénoménologie..., op. cit., p. 15.

31 Merleau-Ponty M., Phénoménologie..., op. cit., p. 183, 204 et 380.

32 Merleau-Ponty M., Phénoménologie..., op. cit., p. 17.
} 
le signe d'une telle prééminence. Ainsi, c'est le partage même du fait et de l'eidos qui se trouve contesté, et en ce sens, il est assurément légitime de dire que Merleau-Ponty réussit là où, aux yeux de Richir, Husserl échoue, à savoir, à " penser tout Wesen comme porté intrinsèquement par la facticité ». Dans la Phénoménologie de la perception, en effet, Merleau-Ponty souligne de manière insistante qu'au niveau d'analyse ouvert par l'étude de la perception, "il n'y a plus aucun moyen de distinguer un plan des vérités a priori et un plan des vérités de fait ", ou qu' " il n'est pas une vérité de raison qui ne garde un coefficient de facticité ${ }^{33}$. Ce brouillage qui fait que « toute vérité de fait est vérité de raison, toute vérité de raison est vérité de fait » est même interprété par Merleau-Ponty comme étant le résultat et l'enseignement ultime de ce «rapport à double sens que la phénoménologie [celle de Husserl, il faut sous-entendre] a appelé Fundierung " ${ }^{34}$ (ce terme renvoyant notamment au rapport de fondation entre l'intuition catégoriale ou l'intuition des idéalités et la perception sensible).

Pourtant, en accordant une place d'honneur au concept de Wesen sauvage, Richir ne nous renvoie pas tant à la Phénoménologie de la perception qu'aux analyses, demeurées en chantier, qui seront publiées dans Le visible et l'invisible ainsi que dans les Notes de travail qui accompagnent cet ouvrage posthume, composite et inachevé. Quelles sont dès lors les avancées supplémentaires que contiennent ces développements tardifs ? Leur point de départ et leur programme ont plus d'une affinité avec les thèses de la Phénoménologie de la perception: Merleau-Ponty exige, ici encore, "que l'on reconsidère la distinction [...] de l'essence et des conditions d'existence ", ou que l'on fasse droit à la "considération du fait ", de « la dimension de facticité », afin de pouvoir maintenir les essences " sous la juridiction des faits $»^{35}$. Mais en même temps, devant la figure inédite de ces "essences brouillées " ${ }^{36}$, il se demande si la question de l'essence est " la question ultime », et si " avec l'essence et le spectateur qui la voit", nous sommes "vraiment à la source $»^{37}$. Le projet du Visible et de l'invisible, notamment dans le chapitre «Interrogation et intuition ", avance dès lors résolument en direction d'une destitution de l'eidétique, en affirmant que «l'être de l'essence n'est pas premier, ne repose pas sur lui-même "; ou, avec plus de force encore, que " des essences que nous trouvons, nous n'avons pas le droit de dire qu'elles donnent le sens primitif de l'être », car

\footnotetext{
Merleau-Ponty M., Phénoménologie..., op. cit., p. 266 et 454.

Merleau-Ponty M., Phénoménologie..., op. cit., p. 454.

Merleau-Ponty M., Phénoménologie..., op. cit., p. 47, 71 et 146.

Merleau-Ponty M., Phénoménologie..., op. cit., p. 146.

Merleau-Ponty M., Phénoménologie..., op. cit., p. 147.
} 
" elles sont le Sosein et non le Sein ${ }^{38}$. L'ontologie du sensible ne s'édifiera pas autour d'une eidétique car l'épreuve primordiale de l'être dans la perception précède la visée ou la saisie de tout eidos.

En outre, dans le cadre de ses dernières recherches, Merleau-Ponty prend davantage conscience de la solidarité secrète qui relie le projet eidétique de Husserl à une conception de la subjectivité dont il essaie de se défaire et que la Phénoménologie de la perception reconduisait encore dans une certaine mesure : comme il l'écrit, « une essence pure qui ne fût pas du tout contaminée et brouillée par les faits ne pourrait résulter que d'un essai de variation totale. Elle exigerait un spectateur sans secrets, sans latence ${ }^{39}$. Ou, pour le dire autrement, puisque «toute idéation [...] se fait dans un espace d'existence ", « il n'y a pas de vision positive qui me donne définitivement l'essentialité de l'essence ${ }^{40}:$ car le succès de la quête eidétique présupposerait, au bout du compte, la transparence et la pleine donation à soi du sujet de la vision et de la variation. Mais la mise au jour de l'opacité et de l'ambiguité de ce dernier, poursuivie inlassablement depuis 1945, révèle l'écart entre l'apparente solidité de l'eidos et son caractère profondément et irrémédiablement "brouillé » ou mobile, et interdit alors de l'envisager « comme une seconde positivité par-delà l'ordre des 'faits' » ou comme le corollaire d'un « impossible travail de l'expérience sur l'expérience qui la dépouillerait de sa facticité comme d'une impureté ${ }^{41}$. Le geste ultime de Merleau-Ponty consiste dès lors à régresser en deçà de la «bifurcation de l'essence et du fait ", vers leur indistinction préalable et primordiale, vers le «milieu de l'existence et de l'essence brutes » au sein duquel, selon une affirmation célèbre du même chapitre "Interrogation et intuition ", " nous n'avons jamais devant nous des individus purs, des glaciers d'être insécables, ni des essences sans lieu et sans date $»^{42}$. Il apparait ainsi qu'en définitive, «les faits et les essences sont des abstractions : ce qu'il y a, ce sont des mondes et un monde et un Être », et « ce monde, cet Être » se caractérisent par leur «facticité et idéalité indivises » ${ }^{33}$. C'est cette indivision du facticiel et de l'idéal au sein du monde sensible qui représente le dernier stade de la refonte merleau-pontienne de l'eidétique, et c'est aussi en vertu d'elle que cette refonte apparaît ultimement comme une destitution.

Or c'est précisément cette indivision de la facticité et de l'idéalité qu'exprime le concept merleau-pontien d'Être sauvage. Il nous faut avouer ici que nous n'avons

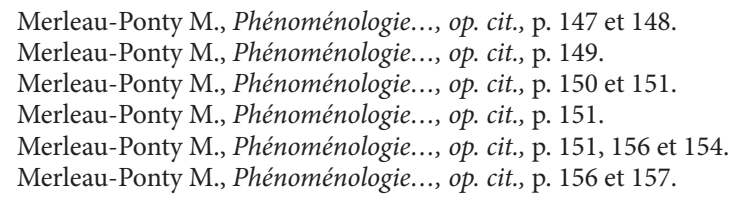


pas réussi à retrouver le concept de Wesen sauvage tel quel sous la plume de Merleau-Ponty (bien que notre enquête se soit limitée aux textes publiés), et cette discrétion s'explique à nos yeux par le fait que l'ontologie indirecte que projette le dernier Merleau-Ponty entend précisément se situer "plus haut que les 'faits', plus bas que les 'essences', dans l'Être sauvage où ils étaient indivis " ${ }^{44}$. Ce niveau ontologique de l'Être brut, synonyme du monde perçu, n'est en effet plus celui de l'essence, et c'est pour cette raison que Merleau-Ponty parle d'Être sauvage plutôt que de Wesen sauvage - ainsi, par exemple, dans une note de travail de janvier 1959 qui contient une ébauche d'introduction pour l'ouvrage qu'il projetait, l'Origine de la vérité, et où il écrit en un style programmatique : « Faire tableau de l'Être sauvage prolongeant mon article sur Husserl » ${ }^{45}$ (à savoir, le célèbre article « Le philosophe et son ombre ", qui paraîtra dans le recueil Signes l'année suivante). Une autre note de travail contemporaine (toujours de janvier 1959) porte pour sa part le titre: "L'Être brut ou sauvage (= monde perçu) ». Cette notation lapidaire nous paraît indiquer aussi un point névralgique de cette voie empruntée par la destitution merleau-pontienne de l'eidétique dans Le visible et l'invisible : l'ontologie indirecte qui prend pour objet l'Être sauvage identifie aussitôt ce dernier au sensible ; en court-circuitant le Wesen au profit du perçu, elle paraît laisser dans l'indécision la dimension de l'idéalité, ou celle du langage. C'est pourquoi l'on peut sans doute considérer que ce n'est pas seulement le projet global du Visible et l'invisible qui a donné l'impulsion la plus significative à la reprise du problème de l'eidétique par Richir, mais aussi les analyses du langage proposées par le dernier Merleau-Ponty et dont on trouve un échantillon remarquable dans le recueil Signes.

Il est certes vrai que, dans Le visible et l'invisible, la considération du Wesen ${ }^{46}$ n'est pas simplement adossée sur le perçu, comme cela paraît en revanche être le cas assez souvent pour l'Être brut ou sauvage. Ainsi, dans une note de travail de février 1960, Merleau-Ponty écrit, de façon encore programmatique : "Montrer qu'il n'y a pas de variation eidétique sans parole; le montrer à partir de l'imaginaire comme soutien de la variation eidétique, et de la parole comme soutien de l'imaginaire ${ }^{47}$. Le langage ne peut manquer de se glisser dans la genèse de l'eidos, et la perception ne peut révéler ce dernier sans le secours de l'imagination. À ce propos,

\footnotetext{
Merleau-Ponty M., Phénoménologie..., op. cit., p. 162.

Merleau-Ponty M., Phénoménologie..., op. cit., p. 219.

46 Nous laissons ici de côté une autre de ses sources incontournables, à savoir la référence manifeste et de plus en plus fréquente au concept heideggérien de Wesen (ou Wesen verbal). Voir par exemple Merleau-Ponty M., Phénoménologie..., op. cit., p. 154, 228 et 280. À ce propos, nous renvoyons à l'étude de Jacques Taminiaux : « Merleau-Ponty lecteur de Heidegger », dans Sillages phénoménologiques. Auditeurs et lecteurs de Heidegger, Bruxelles, Ousia, 2002, p. 273-293, p. 284 en particulier.

47 Merleau-Ponty M., Phénoménologie..., op. cit., p. 290.
} 
les développements publiés dans Signes, tout en prônant à leur tour « le va-et-vient entre [les essences] et les faits » et en invitant à " contester les essences par les faits et les faits par les essences " 48 , ont l'intérêt et le mérite de souligner avec force que "la genèse du sens n'est jamais achevée " ${ }^{49}$, et ce, dans une perspective qui place l'émergence du langage plus résolument au centre de la réflexion. Ces analyses ne se contentent donc pas d'explorer " la logique allusive du monde perçu " ${ }^{50}$, ou le " 'logos du monde esthétique' dont parlait déjà la Logique formelle et transcendantale », mais inscrivent la considération de l' « enveloppement réciproque ${ }^{51} \mathrm{du}$ fait et de l'essence dans l'horizon plus large de la genèse du sens (ou du «devenir $d u$ sens », du « devenir sens » dont parle par ailleurs La prose du monde $e^{52}$ ). Dans cet esprit, Merleau-Ponty souligne le fait que «Husserl [...] change [...] la vision des essences en une 'phénoménologie de la genèse' » et qu'en suivant cette voie la pensée philosophique " ne croit pas tant à des essences qu'à des nœuds de signification qui seront défaits et refaits autrement dans un nouveau réseau du savoir et de l'expérience ${ }^{53}$. C'est à la lumière de tous ces éléments, finalement, que l'article « Le philosophe et son ombre " peut soutenir que « Husserl réveille un monde sauvage et un esprit sauvage ${ }^{54}$. Implicitement, c'est l'inachèvement ou l'incomplétude de l'eidos, ainsi que sa genèse sans terme, qui se trouvent ainsi accentués. Ces indications dessinent quelques-unes des directions les plus importantes qu'empruntera la reprise par Richir de la question eidétique, dont notamment l'articulation du langage et de l'imagination (ressaisie radicalement comme phantasia).

\section{La refonte richirienne de l'eidétique}

Nous aimerions en effet, dans le dernier temps de notre enquête, relever et discuter quelques-unes des avancées les plus enrichissantes de la pensée richirienne du Wesen sauvage, en nous appuyant tout d'abord sur les perspectives esquissées dans Phénomènes Temps et Êtres (1987), mais surtout sur L'institution de l'idéalité (2002) et les "Compléments et corrections à l'Institution de l'idéalité » publiés en 2008 dans les Fragments phénoménologiques sur le langage. Il convient pourtant,

\footnotetext{
Merleau-Ponty M., Signes, Paris, Gallimard, 2001 [1960], p. 230.

Merleau-Ponty M., Signes, op. cit., p. 67.

50 Merleau-Ponty M., Signes, op. cit., p. 92. Voir aussi Merleau-Ponty M., La prose du monde, Paris, Gallimard, 2008 [1969], p. 91, où il est question de la « logique allusive du monde » tout court.

Merleau-Ponty M., Signes, op. cit., p. 171 et 166.

2 Merleau-Ponty M., La prose du monde..., op. cit., p. 178.

3 Merleau-Ponty M., Signes, op. cit., p. 254 et 230.

54 Merleau-Ponty M., Signes, op. cit., p. 294.
} 
avant d'examiner ces analyses, de poser une question qui nous parait importante, à savoir celle de savoir quelle est la portée exacte de l'adjectif "sauvage ", qui se fait de plus en plus présent chez Merleau-Ponty à la fin des années cinquante et que Richir réutilisera à son compte. (Pour un penseur post-merleau-pontien, cet adjectif ne peut pas manquer d'évoquer par ailleurs Lévi-Strauss qui, significativement, dédie à Merleau-Ponty sa Pensée sauvage, parue en 1962, un an après la mort du philosophe.) On en trouve une explicitation et une justification précieuses dans l'article "Monde et phénomène " (1992), contemporain des Méditations phénoménologiques. Richir y soulève déjà la question des «Wesen sauvages eux-mêmes, plus archaïques que l'étant », " échappant à toute téléologie et à toute intentionnalité », et décrits plus loin encore comme des « existentiaux incarnés » et comme des « rayons ou horizons du monde ${ }^{55}$, formules qui ne sont pas sans rappeler directement Merleau-Ponty. Pour Richir, c'est donc vers un registre archaïque, pré-objectif comme pré-ontologique ${ }^{56}$, que fait signe l'adjectif « sauvage » quand il qualifie les Wesen. Ce dernier terme, d'ailleurs, ne renvoie pas toujours au domaine de l'eidos ou de l'idéalité, mais, comme le même article de 1992 l'indique, est pris parfois aussi dans sa signification sans doute première, que l'on rend en français, faute de mieux, par le terme " être ", quand on traduit par exemple Lebewesen par " être vivant ». Dans ce qui suit, cependant, nous allons nous concentrer sur des contextes où la référence aux Wesen sauvages surgit au sein de développements portant sur l'eidos et la dimension de l'idéalité.

Nous n'avons pas la prétention de fournir ici un exposé systématique (ou «architectonique » en ce sens précis) de ces analyses ${ }^{57}$, car cela déborderait largement les cadres de notre étude, mais nous contenterons de dresser un bilan des éléments les plus significatifs qui déplacent et infléchissent la thématisation de l'eidos, toujours dans l'horizon de l'articulation entre facticité et idéalité. Que la référence à Merleau-Ponty soit ici au moins aussi importante que celle à Husserl, c'est ce dont témoigne la première section de Phénomènes Temps et Êtres, qui traite des essences et de l'intuition des essences chez le dernier Merleau-Ponty (dont l'approche

55 Richir Marc, « Monde et phénomènes », Les Cahiers de Philosophie, n 15-16/1992 : « Le Monde », p. 111-137, p. 131.

56 Voir en ce sens Schnell Alexander, Le sens se faisant. Marc Richir et la refondation de la phénoménologie transcendantale, Bruxelles, Ousia, 2011, p. 76.

57 Nous en trouvons un aperçu synthétique au sein de l'ouvrage de Gondek Hans Dieter et Tengelyi László, Neue Phänomenologie in Frankreich, Berlin, Suhrkamp, 2011, p. 50sq. Les auteurs soulignent, tout d'abord, que la transformation du concept de phénomène opérée par Richir va de pair avec une conception métamorphosée de l'eidétique (p. 50), et qu'une alternative à la méthode eidétique husserlienne est trouvée en tirant profit de la réflexion tardive de Merleau-Ponty sur les essences sauvages (p. 51). 
apparaît ainsi comme un préalable au " dépassement de l'eidétique husserlienne ", qui ne sera engagé que plus loin, au sein de la deuxième section de l'ouvrage ${ }^{58}$ ). En effet, en tant que penseur de l'indivision entre factualité et idéalité, l'auteur du Visible et l'invisible est le plus à même de rappeler que «ni l'essence ni le fait ne sont donnés dans le sens de la coïncidence » et, ce faisant, d'amender profondément le projet eidétique husserlien sans le rendre pour autant caduc. Cela revient à considérer, comme le souligne Richir, que « ce qu'il y a d'illusoire dans la Wesenschau husserlienne, n'est pas tant le fait qu' elle intuitionne quelque chose que le fait qu'elle croit l'intuitionner en coïncidence. Et pareil pour ce qui serait une prétendue intuition pure des faits ${ }^{59}$. Encore une fois, la supposée pureté de l'eidos apparait comme le reflet de l'illusoire transparence à soi du sujet de la vision, et les deux appellent une destitution conjointe.

À travers Merleau-Ponty, il s'agit néanmoins pour Richir également de poser la "question [...] de l'origine phénoménologique des essences » et, à cette fin, de faire ressortir la « distorsion originaire du phénomène », qui caractérise en propre sa phénoménalité en tant qu'elle est irréductible à ce qui en est donné actuellement et en tant qu'il est "plus vieux, en son archaïsme primordial, que nos catégories du fait et de l'essence $»^{60}$. Et puisque la distinction du factuel et de l'essentiel nécessite d'être saisie comme secondaire et dérivée, "l'origine phénoménologique de la variation eidétique " et des essences qui en résultent doit être renvoyée à la " 'viscosité" du champ des phénomènes ", que le dernier Merleau-Ponty avait su faire ressortir tout particulièrement. Ainsi, selon Richir, « les essences charnelles, comme rayonnement d'être, ne constituent pas le noyau dur ou ultime des phénomènes, mais autant d'entrées multiples et provisoires dans le monde et les mondes »; autrement dit : «Elles sont [...] autant de pivots où s'accouplent la chair du corps et la chair des mondes, et dont il serait illusoire de chercher la carte ou le plan. »61 Il n'y a donc pas d'eidos pur, épuré de toute facticité, ou désincarné, et même, la chair irréductible de tout eidos n'est pas seulement celle de notre monde (le monde perçu si cher à Merleau-Ponty), car ce monde doit être lui-même soumis à la variation et à la pluralisation.

Nous ne reviendrons pas ici sur les éléments d'analyse qui avaient déjà été déterminants dans le commentaire de Husserl dans l'Appendice aux Méditations phénoménologiques et que L'institution de l'idéalité de 2002 remet à l'honneur, à savoir : le rappel de la «substitu[tion] à la notion d'intersubjectivité transcendantale [de]

\footnotetext{
Richir Marc, Phénomènes Temps et Êtres, Grenoble, Millon, 1987, p. 234sq.

Richir M., Phénomènes..., op. cit., p. 77.

Richir M., Phénomènes..., op. cit., p. 71, 80 et 85.

61 Richir M., Phénomènes..., op. cit., p. 89 et 102-103.
} 
celle d'interfacticité transcendantale ${ }^{62}$, la mise au jour du caractère proprement transcendantal de l'eidétique ${ }^{63}$ et de la " transposition architectonique ${ }^{64}$ qui en découle, ainsi que le rappel du caractère «flottant» ou "clignotant» des Wesen, qualifiés toujours de "sauvages " pour cette raison même ${ }^{65}$. Nous préférons y repérer aussi des avancées nouvelles, qui nous paraissent avoir trait à deux choses particulièrement significatives : d'une part, à l'accentuation du « flou eidétique » en tant qu'irréductible et en tant que lié à une indétermination toujours résiduelle ; et d'autre part - mais les deux aspects sont liés - à l'insistance sur ce que Richir appelle « les infinis eidétiques ${ }^{66}$. Encore, une fois, c'est comme si le plan de l'eidétique et son assise mondaine se trouvaient eux-mêmes démultipliés et complexifiés. Ainsi, d'une part, «il n'y a pas d'eidos sans un flou eidétique qui 'bouge' ou qui 'tremble' dans les lacunes de la continuité »; et de même, «il n'y a pas [...] d'eidos sans une double infinité, due à la prise en compte de la phantasia que nous faisons, mais que Husserl a manquée : celle de l'inépuisable et celle de l'indéterminable "67. Ce double extrait insiste tout d'abord sur l'impossibilité d'arrêter l'eidos, de le fixer, de le déterminer parfaitement et une fois pour toutes, et donc sur la nécessité de tenir compte de sa mobilité et de son indétermination foncières; mais il ouvre aussi vers la question de l'infinité eidétique tout en dévoilant son assise, à savoir la prise en compte radicale de la phantasia qui élargit et approfondit le spectre de la variation. À rebours de la limitation de l'imagination (encore récurrente chez Husserl) à une instance de présentification de l'absent par mise en image, il faut reconnaitre au contraire, comme le propose Richir dans L'institution de l'idéalité, "l'infinité du champ de l'imagination et la pluralité des mondes eidétiques ${ }^{68}$. Cet élargissement et cette démultiplication sont aussi le corollaire de l'impossibilité d'une détermination complète de l'eidos, ou encore d'une vision définitive, d'une Wesenschau sans reste livrant une essence pure et compacte. La percée importante tient ici à la transposition de l'eidétique, comprise préalablement comme eidétique de ce monde, vers des Wesen qui sont ceux « des mondes pluriels qui sont déjà transpossibles par rapport à telle phase de monde ${ }^{69}$; et dans la dimension du

62 Richir Marc, L'institution de l'idéalité, Beauvais, Mémoires des Annales de Phénoménologie, 2002, p. 19.

63 Voir Richir M., L'institution..., op. cit., p. 78 : «l'eidétique transcendantale présuppose l'épochè phénoménologique la plus radicale », et p. 81 pour la référence nécessaire à la Vermöglichkeit subjective expliquant le fait que l'eidétique soit transcendantale.

64 Richir M., L'institution..., op. cit., p. 78.

65 Voir Richir M., L'institution..., op. cit., p. 179 : « des Wesen sauvages [...] qui flottent [...] dans le clignotement».

66 Richir M., L'institution..., op. cit., p. 23.

67 Richir M., L'institution..., op. cit., p. 14.

68 Richir M., L'institution..., op. cit., p. 88.

69 Richir M., L'institution..., op. cit., p. 189. 
transpossible ${ }^{70}$, nous nous situons même «par-delà toute eidétique $»^{71}$. L'eidos outrepasse ainsi le simple registre des possibilités de ce monde, ouvert par des actes de variation qui présupposent toujours celui-ci comme cadre. En même temps et plus loin encore, la démultiplication des mondes rendue possible par la phantasia non seulement élargit le domaine eidétique, mais menace aussi de le faire éclater.

Quels seront dès lors les correctifs et compléments apportés à cette description de l'eidos dans les Fragments phénoménologiques sur le langage (2008) ? Nous prendrons ici pour guide cet extrait où ils nous semblent se trouver remarquablement concentrés : "le champ eidétique est à jamais inchoatif et indéfini dans l'absolu; il est inaccessible, comme l'avait vu Kant, dans sa totalité ; il est donc, dans sa totalité (supposée), infigurable (undarstellbar), et toute eidétique n’est jamais que partielle et locale, ce qui est une autre manière de dire que le flou eidétique est irréductible ${ }^{72}$. En un sens, par rapport aux avancées que nous venons de mettre en avant à partir de L'institution de l'idéalité, nous n'avons pas ici de correctif au sens d'une retouche mais plutôt au sens d'une touche plus appuyée : c'est toujours l'indétermination et la mobilité foncière de l'eidos qui se trouvent soulignées, pour insister cependant davantage sur ce revers de la démultiplication qu'est l'impossibilité de la totalisation ou de l'unification, de la réduction à l'unité du champ eidétique ${ }^{73}$. Il s'agit ce faisant de rompre de manière encore plus nette avec la prétention métaphysique, que Husserl et Heidegger ont reconduite chacun à sa façon, d'une omnitudo possibilitatis, d'une totalisation du possible (fût-elle opérée au sein du monde ou au sein de l'ego) qui aspirerait à livrer un inventaire complet et exhaustif des eidè : contre toute prétention à (ou tentation de) la totalisation, il faut accepter que le « réseau eidétique » reste nécessairement « inchoatif et indéfini, c'est-à-dire [qu']on ne peut pas faire le compte de toutes les possibilités " ${ }^{74}$. Pour exprimer cette inchoativité permanente de l'eidos, Richir va réinvestir et transposer le concept cartésien de «création continuée » afin de décrire «le parcours de la variation $»^{75}$ - de la variation eidétique censée aboutir à la production de l'eidos. Mais la création continuée ne sert pas ici à fournir une assise métaphysique solide

70 Nous ne pouvons pas nous attarder ici sur ce concept important que Richir emprunte à Maldiney, mais nous permettons de renvoyer à l'analyse que nous en avons proposée dans « Du possible au transpossible. Maldiney lecteur et critique de Heidegger» (Philosophie, vol. 130, 2016, p. 58-71).

72 Richir Marc, Fragments phénoménologiques sur le langage, Grenoble, Millon, 2008, p. 160-161.

73 Ce manque d'unité est aussi celui du champ phénoménal, composé de "phénomènes discrets » (pour citer Florian Forestier, qui ajoute en note : “C'est entre autres pour éclaircir cette question de la discrétion que Richir introduit le concept des "Wesen sauvages" " (La phénoménologie génétique de Marc Richir, p. 73, note 35).

74 Richir M., Fragments, op. cit., p. 161.

75 Richir M., Fragments, op. cit., p. 162. 
à l'instabilité ontologique ou eidétique ; son sens est résolument détourné pour exprimer, non pas l'entrée en présence perpétuellement renouvelée, mais plutôt son impossibilité : «L'eidos n'est pas en présence, et n'est pas présent (il n'est pas l'objet d'une intentionnalité) : il est à l'instant, se crée à tout instant " ${ }^{76}$. La création continuée exprime donc elle aussi le caractère toujours inchoatif et jamais achevé de l'eidos, aux antipodes de toute prétention à affirmer sa pureté, sa solidité et sa netteté.

Avant de clore cette analyse, il convient de souligner la remarquable pérennité, sous la plume de Richir, de cette description de l'eidos qui met constamment en avant sa genèse à jamais inachevée, son indétermination irréductible, ainsi que son caractère foncièrement intotalisable, éclaté et pluriel. Elle se trouve, en effet, déjà préfigurée, dans des termes légèrement différents, dans l'article de 1970 « Le rien enroulé - Esquisse d'une pensée de la phénoménalisation (note préliminaire) », où nous pouvons lire : "Wesen n'est ni présence, ni absence, c'est l'élément flottant, situé partout et nulle part, qui inquiète la pensée et la met en mouvement ${ }^{77}$. Et elle revient aussi, de manière significative, dans les conversations avec Sacha Carlson publiées en 2015 sous le titre L'écart et le rien, qui ne manquent pas de rendre à leur tour ${ }^{78}$ hommage à la double impulsion husserlienne et merleau-pontienne qui a mis en mouvement le projet phénoménologique richirien et animé sa reprise critique de l'eidétique ${ }^{79}$. C'est cette double impulsion et quelques-uns de ses effets et prolongements que nous avons tâché de restituer ici.

Claudia Serban est agrégée de philosophie, docteur de l'Université Paris-Sorbonne et ancienne pensionnaire de la Fondation Thiers. Depuis 2015, elle est Maître de conférences à l'Université Toulouse 2 Jean Jaurès. Le livre issu de sa thèse de doctorat, intitulé Phénoménologie de la possibilité : Husserl et Heidegger, est paru en 2016 aux Presses Universitaires de France, dans la collection « Épiméthée ». Elle est également l'auteur de nombreux articles portant sur la phénoménologie allemande et française, ainsi que sur la philosophie allemande classique.

76 Richir M., Fragments, op. cit., p. 162.

77 Richir Marc, «Le rien enroulé - Esquisse d’une pensée de la phénoménalisation (note préliminaire) », Textures, vol. 7-8 (1970), p. 3-24, p. 13-14. Nous devons cette référence au travail de Sabin Bogdan Ivan, et nous tenons à le remercier ici.

78 Richir Marc, L'écart et le rien. Conversations avec Sacha Carlson, Grenoble, Millon, 2015. Voir, notamment, p. 164-165, à propos des Wesen sauvages, ainsi que p. 240 sur l'interfacticité.

79 La reprise critique de l'eidétique n'est toutefois qu' un préambule de la nouvelle élaboration phénoménologique proposée par Richir. Comme le note Florian Forestier au terme de son étude : « Au cours de son développement, la phénoménologie génétique de Richir se comprend d'abord comme une phénoménologie des Wesen sauvages, puis de l'interprétation concrète que l'on peut donner de ce concept encore formel » (La phénoménologie génétique de Marc Richir, p. 194). 